\title{
Aerobic vs Resistance Exercise-An Endocrine Perspective
}

\author{
Narendra Kotwal ${ }^{1}$, Naresh Bansal ${ }^{2}$, Sandeep Kumar ${ }^{3}$
}

\begin{abstract}
Exercise elicits a wide variety of hormonal responses critical for metabolism and consequent tissue growth and development. It is imperative to understand that when we exercise there are acute endocrine responses and subsequent chronic endocrine conditioning. The acute response depends upon the frequency, intensity, and duration of physical exercise. Chronic endocrine conditioning is attained after a long duration of exercises and the magnitude of this conditioning is dependent on persistence, duration, attainment of neuroendocrine responses, and skeletal muscle response which releases myokines. There is ample amount of evidence now to believe muscle as an endocrine organ. Exercise activates various molecular mechanisms in the muscle which alters various endocrine and metabolic milieu inside the muscle. This review aims to provide evidence-based narration of peculiar anabolic and catabolic hormonal responses to exercise and revisit the mechanistic explanation of this occurrence. This review has analyzed the differential endocrine adaptation in aerobic and anaerobic exercises.

Keywords: Aerobic exercise, Exercise endocrinology, Exercise prescription, Overtraining, Resistive exercise.

Journal of Medical Academics (2020): 10.5005/jp-journals-10070-0057
\end{abstract}

\section{INTRODUCTION}

The physiological and psychological advantages of regular exercise are well known for ages. Exercise elicits a wide variety of hormonal responses critical for metabolism and consequent tissue growth and development. The acute hormonal response is dependent on frequency, intensity, and duration while long-term changes in the endocrine system are a function of persistence, duration, and development of neuroendocrine responses. These neuroendocrine responses change receptor content and binding proteins which in turn alters various endocrine and metabolic responses. ${ }^{1}$ There is ample amount of evidence now to believe muscle as an endocrine organ. Exercise activates various molecular mechanisms in the muscle which alters various endocrine and metabolic milieu inside muscle. ${ }^{2}$ This review aims to provide evidence-based narration of peculiar anabolic and catabolic hormonal responses to exercise and revisit the mechanistic explanation of this occurrence. The review was prepared after searching online databases such as PubMed and Google Scholar. Keywords used for the search were exercise and endocrine, exercise and molecular changes, aerobic and resistive exercises, and sports endocrinology. This review has analyzed the differential endocrine adaptation in aerobic and anaerobic exercises (Flowchart 1). ${ }^{3,4}$

\section{Definition of Exercise}

Physical activity is defined as any body movement produced by skeletal muscles that result in energy expenditure beyond resting expenditure while exercise is defined as physical activity that is planned, structured, repetitive, and purposeful and is usually aimed at improving or maintaining physical fitness. The exercise can be isotonic or isometric, either of which can be performed aerobically or anaerobically based on usage of energy, intensity, duration, repetition, and frequency. ${ }^{5}$

Aerobic exercise involves repetitive and continuous movements of large muscles (legs, shoulders, chest, and arms) using oxygen as a means of energy, is critical to losing fat and enhancing cardiovascular and respiratory fitness, and involve activities like walking, running, swimming, cycling. The resistive exercises involve short but high-intensity bursts of physical activity independent of
${ }^{1}$ Armed Forces Clinic, New Delhi, India

${ }^{2}$ Department of Endocrinology, Army Hospital RR, Delhi Cantonment, New Delhi, India

${ }^{3}$ Department of General Medicine, Army College of Medical Sciences, Delhi Cantonment, New Delhi, India

Corresponding Author: Sandeep Kumar, Department of General Medicine, Army College of Medical Sciences, Delhi Cantonment, New Delhi, India, Phone: +91 8427605636, e-mail: sandeep.sapehia@gmail. com

How to cite this article: Kotwal N, Bansal N, Kumar S. Aerobic vs Resistance Exercise-An Endocrine Perspective. J Med Acad 2020;3(1):7-10.

Source of support: Nil

Conflict of interest: None

oxygen. Resistive exercises involve activities like weight lifting and it increases the number of insulin receptors and its insulin sensitivity. It also improves muscle endurance and strength, increasing lean muscle mass and metabolic rate. ${ }^{6}$ Table 1 summarizes various endocrine adaptations in aerobic and resistive exercises. ${ }^{7}$

\section{HORMONES IN EXERCISE}

Exercise regulates cellular metabolism by inducing secretory activity of certain hormones [e.g., adrenocorticotrophic hormone (ACTH), cortisol], facilitating the cardiovascular response, hormone transport across cell membranes (e.g., insulin). Exercise also stimulates energy production pathways and mobilize energy substrates. Hormones are intimately involved with protein synthesis and degradation mechanisms that are part of muscle adaptations to resistance exercise. ${ }^{7,8}$

The other functions of hormones during exercise are the regulation of blood plasma and glucose and fat metabolism. The blood plasma volume is maintained by renin, angiotensin, and aldosterone axis and antidiuretic hormone which help in maintaining blood supply to active tissue. These adaptations ensure increased oxygen-carrying capacity and replenish water for the production of sweat. The blood glucose during exercise 
Flowchart 1: Exercise associated endocrine response. ${ }^{3,4} \mathrm{ACTH}$, adrenocorticotrophic hormone; GH, growth hormone

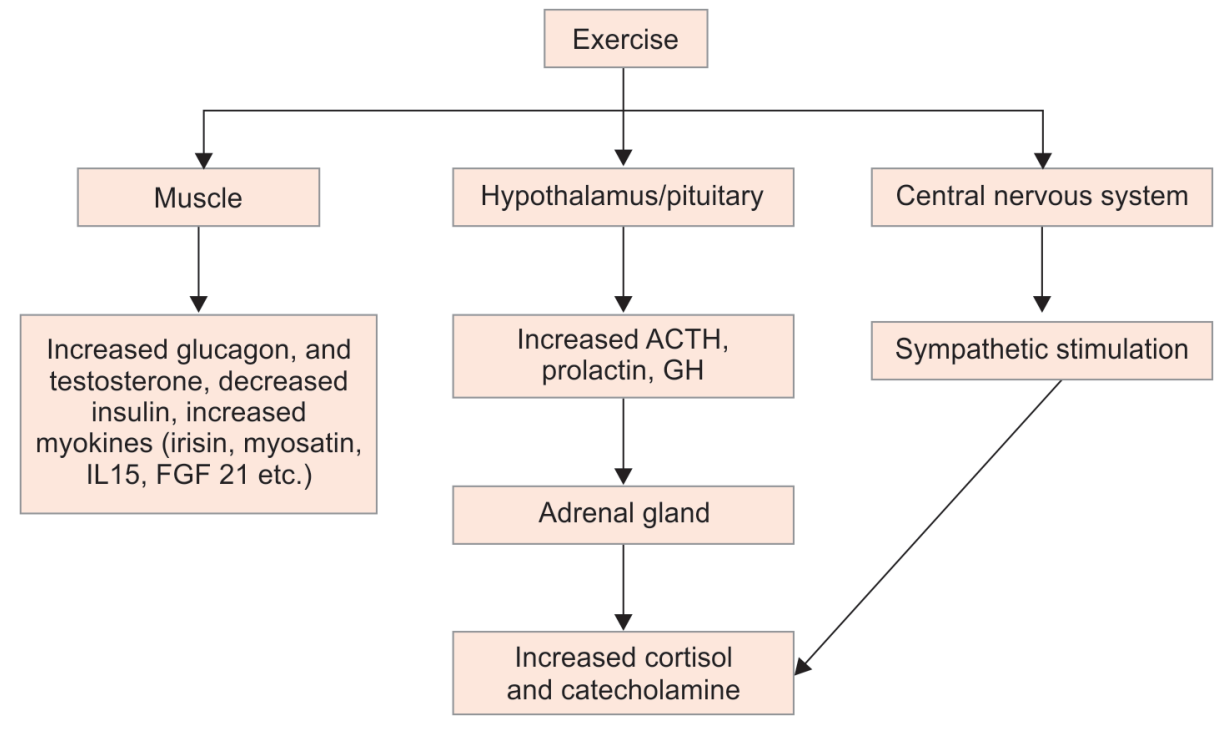

is affected by dietary intake, the liver release of glucose, the muscle uptake and fat metabolism during long-term exercise and is controlled by insulin during rest and glucagon, epinephrine, norepinephrine, cortisol, and growth hormone $(\mathrm{GH})$ during exercise. ${ }^{9}$ The other physiological cascades affected during exercise include an increase in mitochondrial density and oxidative metabolism, increased capillary density, protein synthesis, and muscle hypertrophy involving thyroid hormone and testosterone. There is a further positive impact on lipid profile, increase in highdensity lipoprotein cholesterol (HDL-C), decreased low-density lipoprotein cholesterol (LDLC), and triglycerides. Exercise affects adipose tissue distribution, increases insulin sensitivity, improves cognitive function, and improves the ability to handle psychosocial stressors which translates into better cardiovascular health. ${ }^{10,11}$ There is improved myocardial $\mathrm{Ca}^{2+}$ sensitivity, reduction in left ventricular hypertrophy, and an increase in $\mathrm{Ca}^{2+}$ reuptake in the sarcoplasmic reticulum in skeletal muscles with beneficial effects on cardiac remodeling, myocardial contractility, and strengthening of skeletal muscles. Exercise also causes a reduction in vasoconstrictor and atherogenic endothelin-I (ET-1) and an increase in vasodilator and antiproliferative Ctype natriuretic peptide (CNP). ${ }^{12}$

A multitude of cellular and molecular events occur during exercise involving a gradual alteration in protein mass and enzyme activities regulated by various hormones. The steroidal and thyroid hormones pass through cell membrane freely and interact with nuclear receptors where it binds with DNA, initiating transcription, and causing protein synthesis. The faster peptide hormones interact with cell membrane receptors and initiate various phosphorylation reactions mediated by second messengers. Besides, various exercise-induced epigenetic changes like the expression of different types of microRNAs, histone modification including methylation and acetylation, and DNA methylation-demethylation occur which play a key role in regulating steroid hormone biosynthesis and in the tissue-specific function of peptide hormones. ${ }^{1}$

The skeletal muscle has also been identified as an endocrine organ capable of releasing small peptides called myokines into the bloodstream which improves the function of the muscles by regulating the intramuscular cross-talk of myofibers, fibroblasts, immune cells, vasculature, and bone. ${ }^{2}$ These include IL-6, IL-8, IL-10,
CC-chemokine ligand (CCL)2, calprotectin S100A9 and vascular endothelial growth factor (VEGF), angiopoietin-like 4 (ANGPTL4), brain-derived neurotrophic factor (BDNF), connective tissue growth factor (CTGF), cysteine-rich angiogenic protein 61 (CYR61), and nicotinamide phosphoribosyltransferase (NAMPT). ${ }^{13}$ These factors act as endocrine signaling mediators and result in beneficial effects of exercise on almost all cell types and organs. ${ }^{14}$

The optimum "exercise prescription" to improve health comprises 20-60 minutes of low- to moderate-intensity aerobic exercise for 4-7 days per week (target of at least 150 minutes per week). The training of $<2$ days per week does not generally result in a meaningful increase in exercise capacity. The resistive exercises for the development of muscle power should include 2-3 sets of $8-12$ repetitions carried out $2-3$ days per week. ${ }^{15}$

If exercise training programs are excessive (intensity, volume, or both), inappropriate or maladaptive hormonal responses may occur characterized by generalized apathy, anorexia, emotional instability, negative nitrogen balance, menstrual disturbances, and increased susceptibility to infections. These occur secondary to elevated levels of steroid hormones, prolactin, and catecholamines, and a decrease in $\mathrm{GH}$, follicle-stimulating hormone (FSH), luteinizing hormone, and testosterone. The excessive local and systemic inflammation mediated by proinflammatory cytokines like IL-1 $\beta$, IL-6, and TNFa acts on multiple levels of hypothalamic pituitary adrenal axis affecting mood, sexual, and additional immune functions. ${ }^{8,16}$

\section{Exercise, Insulin, and Diabetes}

Insulin is a potent anabolic hormone and its serum concentration parallels changes in blood glucose and depends on the ingestion of nutrients before and during exercise. Exercise alters the blood glucose homeostasis depending on the type, intensity, and duration of exercise and the pre-exercise blood glucose levels. Persistent aerobic exercise increases the risk of hypoglycemia during and postexercise while resistive exercise increases the blood glucose level initially due to the release of counter-regulatory hormones followed by hypoglycemia as their levels decrease. However, both types of exercise improve insulin sensitivity and lower blood glucose. This has an important clinical role in preventive and curative strategies in metabolic syndrome and type II diabetes. ${ }^{17}$ The fasting plasma 
Table 1: Comparison of endocrine adaptation in aerobic vs resistive exercises

\begin{tabular}{lll}
\hline Endocrine/metabolic & Aerobic exercise & Resistive exercises \\
\hline adaptations & $\uparrow$ & $\uparrow \uparrow$ \\
Quality of life & $\uparrow \uparrow$ & $\uparrow$ \\
Buscle strength/muscle mass & $\leftrightarrow$ & $\uparrow$ \\
Glycemic control & $\leftrightarrow$ & $\uparrow$ \\
Insulin signaling & $\uparrow$ & $\uparrow$ \\
Immune function & $\uparrow$ & $\uparrow$ \\
HDL-C & $\uparrow$ & $\uparrow$ \\
LDL-C & $\downarrow$ & $\downarrow$ \\
Triglycerides & $\downarrow$ & $\downarrow$ \\
ACTH/cortisol & $\uparrow$ & $\uparrow$ \\
Aldosterone & $\uparrow$ & $\uparrow$ \\
$\beta$-Endorphin & $\uparrow$ & $\uparrow$ \\
Epinephrine & $\uparrow$ & $\uparrow \uparrow$ \\
Estrogen & $\uparrow$ & $\uparrow$ \\
FSH/LH & $\downarrow$ & $\leftrightarrow \downarrow$ \\
GH & $\uparrow$ & $\uparrow \uparrow$ \\
Prolactin & $\uparrow$ & $\uparrow$ \\
Testosterone & $\uparrow$ & $\uparrow \uparrow$ \\
T4 & $\uparrow$ & $\uparrow$ \\
T3 & $\downarrow$ & $\downarrow$ \\
TSH & $\leftrightarrow$ & $\leftrightarrow$ \\
\hline ACTH a & $\uparrow$ & $\uparrow$
\end{tabular}

$\mathrm{ACTH}$, adrenocorticotrophic hormone; $\mathrm{FSH}$, follicle-stimulating hormone; $\mathrm{GH}$, growth hormone; HDL-C, high-density lipoprotein cholesterol; LDL-C, low-density lipoprotein cholesterol; LH, luteinizing hormone; TSH, thyroidstimulating hormone

glucose is more significantly affected by resistance training than aerobic exercise while postprandial hyperglycemia, a marker of decreased endothelial function, increased oxidative stress, and inflammation is improved by the combined training group. However, both aerobic and resistance training can increase insulin sensitivity, improve abnormal lipid profile and visceral adiposity by recruiting more skeletal muscle fibers with enhanced glycogen storage capacity and expression of additional glucose transporters (GLUT4). ${ }^{10}$ Insulin has a potent upregulating effect on muscle protein synthesis with ingestion of carbohydrates and amino acids before or during resistance exercise is especially important to maximize this protein synthesis. The beneficial effects of exercise are seen with respect to cardiovascular risk factors in diabetes like changes in endothelial function, carotid artery intima-media thickness, left ventricular ejection fractions, and improvements in exercise capacity. 9,14

\section{EXERCISE AND TESTOSTERONe}

Resistance exercise results in an increase in the blood concentrations of anabolic hormones. The primary androgen hormone, testosterone, increases in response to sympathetic stimulation and plasma volume reduction. This is more marked in men than in women (androgen remains mostly unchanged). ${ }^{18}$ This testosterone, upon secretion, interacts with skeletal muscle tissue and causes protein synthesis, increased strength, size of skeletal muscle, and increased force production potential and muscle mass. ${ }^{4}$ The effects have also formed the basis of the use of precursors of testosterone (i.e., $300 \mathrm{mg}$ of androstenedione $+150 \mathrm{mg}$ of DHEA) as drugs of abuse in sports. Aerobic exercises in women with polycystic ovarian disease (PCOS) offer significant benefits and are an integral part of PCOS management. However, extremely high-intensity resistive exercises may be counterproductive in PCOS by causing delayed menarche, amenorrhea, and anovulation by decreasing the luteinizing hormone (LH) levels. ${ }^{19}$

\section{Exercise and Growth Hormone}

Growth hormone affects the target tissues like liver tissue, skeletal muscle, fat cells, and bone. The impact of GH is in coordination with other neuroendocrine feedback mechanisms and is regulated by other secondary hormones. Like testosterone, GH increases significantly in response to high-intensity resistive exercises. Growth hormone decreases glucose utilization and glycogen synthesis which helps in maintaining apt glucose homeostasis during exercises. Growth hormone also facilitates amino acid transport across cell membranes and increases protein synthesis. It enhances lipolysis and promotes the utilization of free fatty acids. Growth hormone supports exercises by stimulating cartilage growth. Exercise also results in acute increases in blood levels of insulin like growth factor 1 (IGF-1) and results in an increase in protein synthesis. ${ }^{20}$

\section{Exercise and Cortisol}

The levels of the adrenal hormone and cortisol, which has predominant catabolic effects, increase with resistive training (stimulates lipolysis in adipose cells and increases protein degradation in muscle cells) but the anabolic effects of $\mathrm{GH}$ and testosterone normally increase to a greater extent and reduce the negative effects of cortisol due to adaptation processes. ${ }^{21}$ The main beneficial action of cortisol during exercise is to protect glycogen stores and shift metabolism to other energy sources. ${ }^{22}$

\section{Exercise and Catecholamines}

The catecholamines especially epinephrine is released during maximal exercise and augments force production in muscles via central mechanisms and increased metabolic enzyme activity for energy generation. It also increases muscle contraction rate and blood flow to it. It enhances secretion rates of other hormones, such as testosterone. ${ }^{9}$ The plasma concentrations of catecholamines are increased by acute resistance exercise and the magnitude of its rise is dependent on muscle groups involved, strength, duration of exercise, and the rest intervals. The "anticipatory rise" is a part of the body's psychological response before the exercise is undertaken. ${ }^{8}$

\section{EXERCISE AND ENDORPHINS}

The levels of endorphins vary during exercise, i.e., rises during exercise and post-exercise reductions may be seen. The increased levels are seen more during resistive exercises than during aerobic exercises. ${ }^{14}$ The acute elevation in $\beta$-endorphins is dependent on muscle mass used, intensity, duration of resistance exercise, and rest intervals. $\beta$-endorphins are implicated in several processes like pain modulation, immune function, and assisting in glucose and lipid homeostasis. They increase the pain threshold in response to exercise training especially resistance training. ${ }^{23}$

\section{Exercise and Thyroid Hormones}

The thyroid hormones, T3 and T4, are important for maintaining the metabolic rate and allowing other hormones to bring about their 
full effect, i.e., the permissive action. However, there is no significant change in levels of thyroid hormones with exercise. ${ }^{24}$ T4 (FT4) increases, T3 (FT3) decreases, and thyroid-stimulating hormone (TSH) remains unaffected in exercise and is more pronounced in calorie deficiency or ultradistance exercise and is reversed by high-calorie intake. ${ }^{25}$ The trained athletes may have increased production and turnover of T4 but baseline thyroid hormone levels do not appear to be affected substantially by chronic exercise (i.e., endurance). ${ }^{26}$

\section{Exercise and Fluid Homeostasis Hormones}

The blood volume critical for fluid homeostasis is maintained by renin, angiotensin, and aldosterone axis and antidiuretic hormone and their levels increase proportionately with exercise intensity, duration, and hydration status of an individual to maintain fluid volume within normal limits. ${ }^{24}$

\section{Exercise and Adipokines}

Adipokines like leptin and apelin are also secreted by adipose tissue and play a role in lipid metabolism, regulation of appetite, and energy homeostasis. ${ }^{11}$

Leptin, a peptide hormone from adipose tissue, relays satiety signals to the hypothalamus to regulate appetite and energy balance, reduces testicular steroidogenesis (both as a direct effect and central by decreasing levels of $\mathrm{LH}$ and FSH). The resistive exercises lead to a reduction in leptin concentrations. ${ }^{8}$

Apelin promotes insulin sensitivity and lipolysis and its levels are directly proportional to insulin levels, total cholesterol, and LDL-C. The levels of apelin decrease after both aerobic and resistance exercise. ${ }^{3,19}$

\section{SUMMARY}

Exercise elicits a range of hormonal responses responsible for cellular metabolism, facilitation of cardiovascular response to exercise and growth, and remodeling of the tissues. Exercise has been shown to improve insulin sensitivity, increase lipid catabolism, improve arterial compliance and endothelial function, and maintain muscle metabolic capacity.

\section{References}

1. Hicks $S D$, Jacob $P$, Middleton $F A$, et al. Distance running alters peripheral microRNAs implicated in metabolism, fluid balance, and myosin regulation in a sex-specific manner. Physiol Genomics 2018;50(8):658-667. DOI: 10.1152/physiolgenomics.00035.2018.

2. Hoffmann C, Weigert C. Skeletal muscle as an endocrine organ: the role of myokines in exercise adaptations. Cold Spring Harb Perspect Med 2017;7(11):a029793. DOI: 10.1101/cshperspect.a029793.

3. Clark A, Mach N. Exercise-induced stress behavior, gut-microbiotabrain axis and diet: a systematic review for athletes. J Int Soc Sports Nutr 2016;13:43. DOI: 10.1186/s12970-016-0155-6.

4. Warren MP, Goodman LR. Exercise-induced endocrine pathologies. J Endocrinol Invest 2003;26(9):873-878. DOI: 10.1007/BF03345238.

5. Caspersen CJ, Powell KE, Christenson GM. Physical activity, exercise and physical fitness definitions for health-related research. Public Health Rep 1985;100(2):126-131.

6. Hawley JA, Lundby C, Cotter JD, et al. Maximizing cellular adaptation to endurance exercise in skeletal muscle. Cell Metabolism 2018;27(5):962-976. DOI: 10.1016/j.cmet.2018.04.014.
7. NCT03200795. Effect of Different Exercises on Musculoskeletal Pain,Glucose Level and Quality of Life Among Patients With Diabetes. https://clinicaltrials.gov/show/NCT03200795. 2017.

8. Ogasawara J, Izawa T, Sakurai T, et al. The molecular mechanism underlying continuous exercise training-induced adaptive changes of lipolysis in white adipose cells. J Obes 2015;2015:473430.

9. Bellavere F, Cacciatori V, Bacchi E, et al. Effects of aerobic or resistance exercise training on cardiovascular autonomic function of subjects with type II diabetes: a pilot study. Nutr Metab Cardiovasc Dis 2018;28(3):226-233. DOI: 10.1016/j.numecd.2017.12.008.

10. Ferrari F, Bock PM, Motta MT, et al. Biochemical and molecular mechanisms of glucose uptake stimulated by physical exercise in insulin resistance state: role of inflammation. Arquivos Brasileiros de Cardiologia 2019;28(3):226-233.

11. Lombardi G, Sanchis-Gomar F, Perego S, et al. Implications of exercise-induced adipo-myokines in bone metabolism. Endocrine 2016;54(2):284-305. DOI: 10.1007/s12020-015-0834-0.

12. Patel H, Alkhawam H, Madanieh R, et al. Aerobic vs anaerobic exercise training effects on the cardiovascular system. World J Cardiol 2017;9(2):134-138.

13. Huh JY. The role of exercise-induced myokines in regulating metabolism. Archives of Pharmacal Research 2018;41(1):14-29. DOI: 10.1007/s12272-017-0994-y.

14. McLeod JC, Stokes T, Phillips SM. Resistance exercise training as a primary countermeasure to age-related chronic disease. Frontiers in Physiology 2019;10:645. DOI: 10.3389/fphys.2019.00645.

15. Garber C, Blissmer B. American college of sports medicine position stand. quantity and quality of exercise for developing and maintaining cardiorespiratory, musculoskeletal, and neuromotor fitness in apparently healthy adults: guidance for prescribing exercise. Med Sci 2011;43(7):1334-1359.

16. Meeusen R, Duclos M, Gleeson M, et al. Prevention, diagnosis and treatment of the overtraining syndrome. ECSS position statement "task force". Eur J Sport Sci 2006;6(1):1-14. DOI: 10.1080/17461390600617717.

17. Carlo Negri EB. Influence of acute bouts of exercise on blood glucose in type II diabetic patients, as measured by continuous glucose monitoring systems. J Diabetes Metab 2013;4(9):1-8.

18. Enea C, Boisseau N, Fargeas-Gluck MA, et al. Circulating androgens in women exercise-induced changes. Sport Med 2011;41(1):1-15. DOI: 10.2165/11536920-000000000-00000.

19. Jang SH, Paik IY, Ryu JH, et al. Effects of aerobic and resistance exercises on circulating apelin-12 and apelin-36 concentrations in obese middle-aged women: a randomized controlled trial. BMC Womens Health 2019;19(1):23. DOI: 10.1186/s12905-019-0722-5.

20. Bonert VS, Melmed S. Growth hormone. In: The Pituitary. 4th edn, 2017.

21. Hötting K, Schickert N, Kaiser J, et al. The effects of acute physical exercise on memory, peripheral BDNF, and cortisol in young adults. Neural Plast 2016;2016:6860573.

22. Zschucke E, Renneberg B, Dimeo F, et al. The stress-buffering effect of acute exercise: evidence for HPA axis negative feedback. Psychoneuroendocrinology 2015;51:414-425. DOI: 10.1016/j. psyneuen.2014.10.019.

23. Hildebrandt $T$, Shope $S$, Varangis $E$, et al. Exercise reinforcement, stress, and $\beta$-endorphins: an initial examination of exercise in anabolic-androgenic steroid dependence. Drug Alcohol Depend 2014;139:86-92. DOI: 10.1016/j.drugalcdep.2014.03.008.

24. Lima LG, Bonardi JTM, Campos GO, et al. Combined aerobic and resistance training: are there additional benefits for older hypertensive adults? Clinics 2017;72(6):363-369. DOI: 10.6061/ clinics/2017(06)06.

25. Ciloglu F, Peker I, Pehlivan A, et al. Exercise intensity and its effects on thyroid hormones. Neuroendocrinol Lett 2005;26(6):830-834.

26. Alkadhi KA. Exercise as a positive modulator of brain function. Mo Neurobiol 2018;55(4):3112-3313. DOI: 10.1007/s12035-017-0516-4. 